\title{
Correction to: Risk Factors for Prognosis in Elderly Patients with Severe Aneurysmal Subarachnoid Hemorrhage: A Retrospective Study
}

\author{
Meng Qi · Lidan Jiang · Yueqiao Xu · Xin Qu · Na Wang • \\ Wenjin Chen · Weitao Cheng · Ning Wang (D)
}

Published online: February 18, 2021

(c) Springer Healthcare Ltd., part of Springer Nature 2021

\section{Correction to: Adv Ther}

https://doi.org/10.1007/s12325-020-01531-7

The original article has published with incorrect dates in the section of patients of methods "A total of 49 patients aged $C 65$ years with severe aSAH (Hunt and Hess [H-H grade III-V) [8] who were admitted to the Department of Neurosurgery of Capital Medical University Xuanwu
Hospital from July 2018 to September 2019 were retrospectively analysed".

The correct sentence is "A total of 49 patients aged $\mathrm{C} 65$ years with severe aSAH (Hunt and Hess [H-H grade III-V) [8] who were admitted to the Department of Neurosurgery of Capital Medical University Xuanwu Hospital from "March 2015 to March 2017" were retrospectively analysed". 